J. Clin. Chem. Clin. Biochem.

Vol. 15,1977 , pp. 603-605

\title{
Reagenziensparende Modifikation der Glucose-Dehydrogenase-Methode für den AutoAnalyzer II zur Glucosebestimmung in Venen- und Kapillarblut
}

\author{
Von K. J. Kornmüller und O. Müller-Plathe
}

Aus dem Zentrallaboratorium (Chefarzt: Dr. O. Müller-Plathe) des Allgemeinen Krankenhauses Altona, Hamburg

(Eingegangen am 1. März/31. Mai 1977)

Zusammenfassung: Es wird eine Modifikation der Glucose-Dehydrogenase-Methode für den AutoAnalyzer II beschrieben, durch die der Verbrauch an Enzymen und Coenzym gegenüber den bisherigen Angaben ohne Einbuße an Linearität beträchtlich reduziert wird. Bei einer Probenfrequenz von 80 pro Stunde gestattet das Fließschema die Glucosebestimmung aus Venenblut und aus verdünnten Kapillarblut-Hämolysaten ohne apparative Umrüstung.

\section{A reagent-saving modification of the glucose dehydrogenase method for the determination of glucose in venous and capillary blood using the Autoanalyzer II}

Summary: A modification of the glucose dehydrogenase method for the Autoanalyzer II is described, which permits a considerable reduction in the quantities of enzymes and coenzyme, without affecting linearity. Using the new modification and a sample frequency of 80 per hour, glucose can be determined in venous blood and in diluted capillary blood haemolysates with no change in the construction of the apparatus.

\section{Einführung}

Zur Bewältigung steigender Analysenzahlen wurde in unserem Laboratorium die Glucose-DehydrogenaseMethode mit dem AutoAnalyzer Il eingeführt. Für diese Methode sprachen die einfache Handhabung, die Haltbarkeit und der relativ niedrige Preis der Reagenzien bei ausreichender Spezifität $(1,2)$, für den AutoAnalyzer sprach die Einsparung eines gesonderten Enteiweißungsschrittes. Der Reagenzienverbrauch sollte dabei weitestgehend vermindert werden. Im folgenden wird nach gut halbjähriger Bewährung in der Routine eine reagenziensparende Modifikation der Glucose-DehydrogenaseMethode beschrieben, mit der die Bestimmung sowohl im Plasma venöser Blutproben wie auch in KapillarblutHämolyṣaten dưrchgeführt werden kann.

\section{Mäterial und Methoden}

\section{Geräte}

AutoAnalyzer II mit Probennehmer IV, Pumpe II, Kolorimeter, Schreiber und Drucker.

In $A$ bbildung 1 ist das von uns verwendete Fließschema dargestellt.

\section{Reagenzien}

1. Pufferlösung pH 7,6, Merck Nr. 1.4051

2. Reaktionslösung

a) Enzymgemisch (Glucose-Dehydrogenase, Mutarotase), Merck Nr. 14055 b) Coenzym (NAD), Merck Nr. 14055

c) Pufferlösung pH 7,6, Merck Nr. 14051. 1 Flasche Enzymgemisch und 1 Flasche Coenzym werden in 1 Liter Pufferlösung gelöst.

Konzentration der Reaktionslösung:

$0,12 \mathrm{~mol} / 1$ Phosphatpuffer $\mathrm{pH} \mathrm{7,6}$

$0,15 \mathrm{~mol} / 1 \quad \mathrm{NaCl}$

2,6 kU/1 Glucose-Dehydrogenase

$55 \mathrm{U} / 1 \quad$ Mutarotase

$0,55 \mathrm{mmol} / 1 \mathrm{NAD}$

Haltbarkeit bei $+4{ }^{\circ} \mathrm{C}$ etwa 4 Wochen

3. NaCl-Brij-Lösung, Merck Nr. 9407.

Probenvorbereitung

Venenblut

EDTA-Kalium und Jodacetat-Natrium enthaltende Probenröhrchen $^{1}$ ) zur Blutentnahme. Gute Durchmischung. Zentrifugation 5 Minuten bei $1500 \mathrm{~g}$; Beschickung der Probenbecher mit $250 \mu \mathrm{l}$ Plasma (ausreichend für 2 Bestimmungen).

\section{Kapillarblut}

Hämolysierlösung ${ }^{2}$ ):

$3 \dot{\text { g }}$ Benzoesäure, Riedel-de Haen Nr. 33045

1 g Natriumfluorid, Merck Nr. 6441

$1 \mathrm{~g}$ Di-Natriumoxalat, Merck Nr. 6556 in 1 Liter dest. Wasser lösen.

Zu $200 \mu l$ Hämolysierlösung als Vorlage in Eppendorfgefäßen gibt man $40 \mu 1$ Kapillarblut. Gut mischen, zentrifugieren. Die

1) Fa. W. Sarstedt, 5223 Nümbrecht-Rommelsdorf.

2) Kompatibel mit dem Glucose-Analyzer der Fa. Yellow Springs Instruments (Vertretung für die Bundesrepublik Deutschland: Kipp \& Zonen, 6242 Schönberg), der bei uns für Notfall-Gluco sebestimmungen eingesetzt wird. 


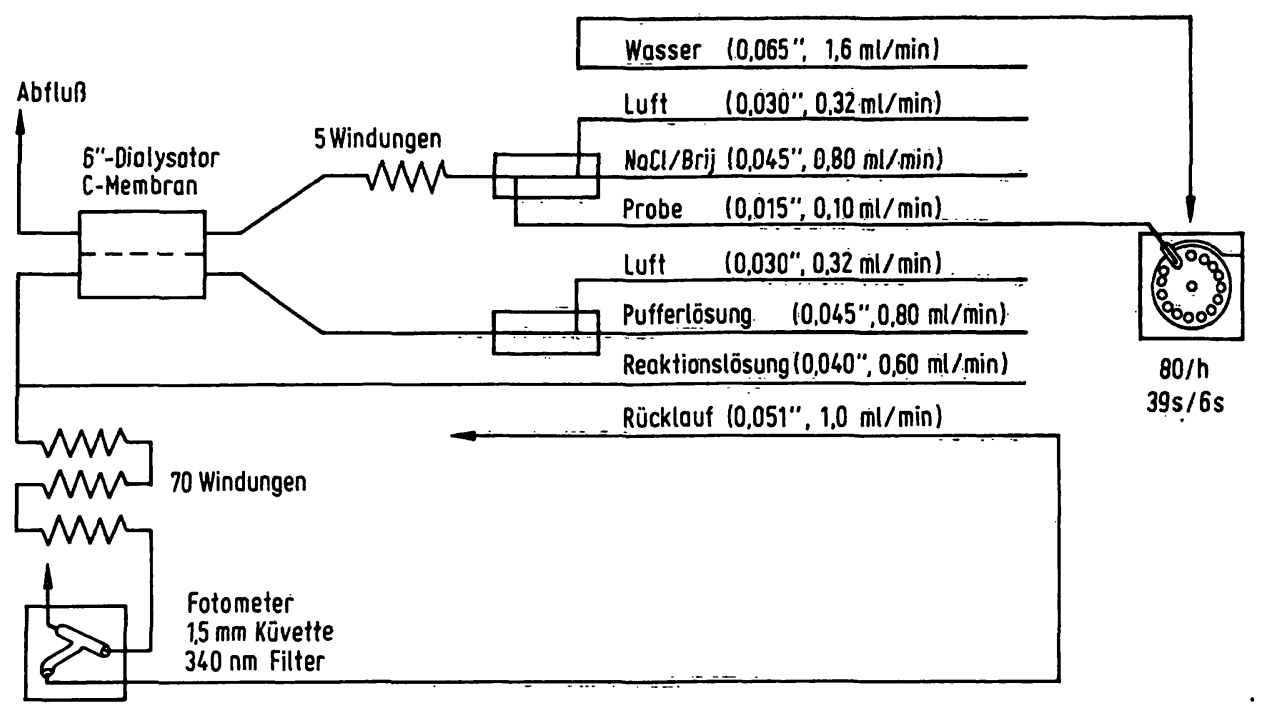

Abb. 1. Fließschema der modifizierten Glucosebestimmung mit der Glucose-Dehydrogenase-Methode für den AutoAnalyzer II.

Gefäße werden mit Hilfe eines Adapters ${ }^{3}$ ) in den Teller des Probennehmers eingesetzt.

\section{Arbeitsweise}

Zur Kalibrierung können wäßrige oder eiweißhaltige Standards verwendet werden. Eine Beeinflussung der Meßergebnisse durch Protein wurde durch vergleichende Untersuchungen in zwei Konzentrationsbereichen ausgeschlossen (Tab. 1).

Zur Analyse von Venenblut wird die Standardlösung unverdünnt eingesetzt. Für Kapillarblut-Hämolysate wird Standardlösung im gleichen Verhältnis wie das Kapillarblut selbst mit physiologischer $\mathrm{NaCl}-\mathrm{Lösung}$ verdünnt. Die Schreiber- und Druckeranzeige wird in beiden Fällen auf den Sollwert eingestellt, so daß auch die Meßwerte der Hämolysate konzentrationsrichtig ausgegeben werden.

\section{Ergebnisse und Diskussion}

\section{Verbrauch an Reaktionslösung}

Die Verminderung des Verbrauches an Enzymen und Coenzym gegenüber den vorliegenden Anleitungen (2, 3 und 4) wird auf zwei Wegen erreicht:

1. Herabsetzung der Durchflußrate an Reaktionslösung auf $0,6 \mathrm{ml} / \mathrm{min}$.

2. Steigerung der Probenfrequenz auf $80 /$ Stunde.

Tab. 1. Einfluß von Protein auf die Glucosebestimmung.

\begin{tabular}{|c|c|c|c|}
\hline \multicolumn{2}{|c|}{$\begin{array}{l}\text { Sollwert } \\
(\mathrm{mmol} / \mathrm{l})\end{array}$} & \multirow{2}{*}{$\begin{array}{c}\begin{array}{l}\text { Ergebnis }(\bar{x} \pm s) \\
(\mathrm{mmol} / \mathrm{l})\end{array} \\
5,56 \pm 0,025 \\
5,58 \pm 0,049\end{array}$} & \multirow{2}{*}{$\begin{array}{l}\mathrm{n} \\
5 \\
5\end{array}$} \\
\hline 5,55 & $\begin{array}{l}\text { in } \mathrm{H}_{2} \mathrm{O} \\
\text { in Albumin }(55 \mathrm{~g} / \mathrm{l})\end{array}$ & & \\
\hline 13,9 & $\begin{array}{l}\text { in } \mathrm{H}_{2} \mathrm{O} \\
\text { in Albumin }(55 \mathrm{~g} / \mathrm{l})\end{array}$ & $\begin{array}{l}13,83 \pm 0,054 \\
13,88 \pm 0,099\end{array}$ & $\begin{array}{l}6 \\
6\end{array}$ \\
\hline
\end{tabular}

3) Fa. Dr. J. Rosenhagen GmbH, Kohfurt 19, 2000 Norderstedt bei Hamburg.
Tab. 2. Reagenzienverbrauch pro Analyse.

\begin{tabular}{|c|c|c|c|c|c|}
\hline \multirow[t]{2}{*}{ Autor } & \multicolumn{2}{|c|}{ Linearitätsbereich } & \multirow{2}{*}{$\begin{array}{l}\text { Glucose } \\
\text { Dehy- } \\
\text { dro- } \\
\text { genase } \\
\text { (U) }\end{array}$} & \multirow{2}{*}{$\begin{array}{l}\text { Mutario- } \\
\text { tase } \\
\text { (mỤ) }\end{array}$} & \multirow{2}{*}{$\begin{array}{l}\text { NAD } \\
(\mu \mathrm{mol})\end{array}$} \\
\hline & $(\mathrm{mmol} / \mathrm{l})$ & (mg/dl) & & & \\
\hline $\begin{array}{l}\text { l. c. (2) } \\
\text { l. c. (3) } \\
\text { l. c. (4) } \\
\text { selbst }\end{array}$ & $\begin{array}{l}\text { bis } 27,8 \\
\text { bis } 33,3 \\
\text { bis } 22,2 \\
\text { bis } 22,2\end{array}$ & $\begin{array}{l}500 \\
600 \\
400 \\
400\end{array}$ & $\begin{array}{r}12,6 \\
2,6 \\
1,7 \\
1,2\end{array}$ & $\begin{array}{r}420 \\
55 \\
60 \\
25\end{array}$ & $\begin{array}{l}1,25 \\
0,55 \\
0,40 \\
0,25\end{array}$ \\
\hline
\end{tabular}

Der Verbrauch an Enzymen pro Analyse im Vergleich mit den Angaben anderer Autoren geht aus Tabelle 2 hervor.

\section{Linearität}

Die Methode erwies sich als linear bis mindestens $22,2 \mathrm{mmol} / 1(400 \mathrm{mg} / \mathrm{dl})$, für praktische Belange bis $27,8 \mathrm{mmol} / \mathrm{l}(500 \mathrm{mg} / \mathrm{dl})$, da Proben dieser Konzentration lediglich um durchschnittlich $2,8 \%$ zu niedrig bestimmt werden.

\section{Präzision}

Die Präzision ist, wie die Tabelle 3 a ergibt, sowohl in der Serie wie auch von Tag zu Tag als gut zu bezeichnen.

Tab. 3. Qualitätskriterien der modifizierten Glucose-Dehydrogenase-Methode.

a) Präzisiọn

\begin{tabular}{lclll}
\hline & $\bar{x}(\mathrm{mmol} / \mathrm{l})$ & $\mathrm{s}(\mathrm{mmol} / \mathrm{l})$ & VK $(\%)$ & $\mathrm{n}$ \\
\hline In der Serie & 4,22 & 0,024 & 0,57 & 9 \\
& 12,65 & 0,037 & 0,29 & 9 \\
Von Tag zu Tag & 4,22 & 0,083 & 1,97 & 75 \\
& 12,65 & $\ldots, 206$ & 1,63 & 75 \\
\hline
\end{tabular}


b) Vergleich mit den Sollwertangaben für die Hexokinase- und Glucose-Dehydrogenase-Methode in mmol/l (mg/dl).

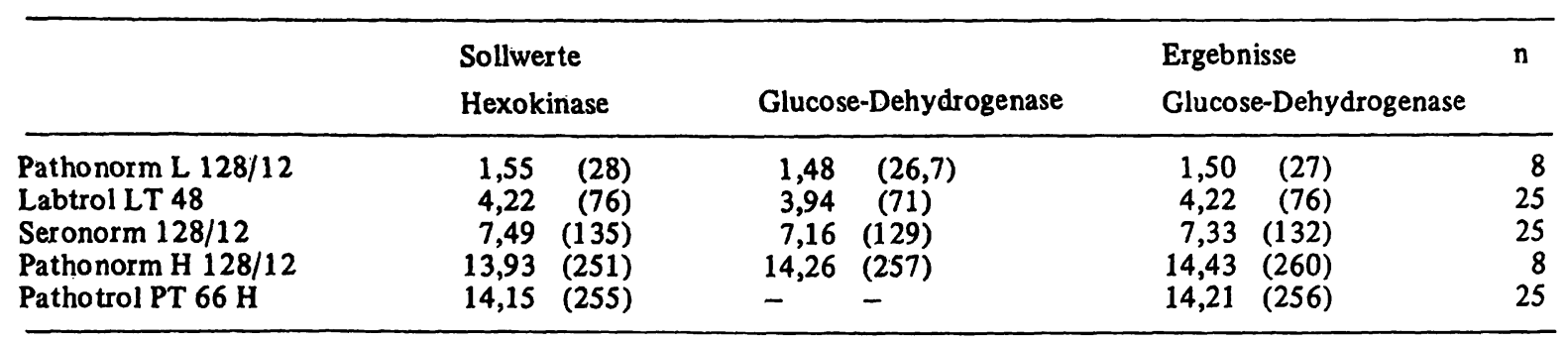

\section{Rich tigkeit}

Tabelle $3 \mathrm{~b}$ ermöglicht einen Vergleich der Sollwertangaben für die Hexokinase- und (soweit vorhanden) für die Glucose-Dehydrogenase-Methode mit unseren Ergebnissen.

Recovery-Versuche an zwei Poolplasmen mit Ausgangskonzentrationen von 7,99 und $6,22 \mathrm{mmol} / \mathrm{l}$ und Zusätzen von 5,0 bzw. $10,0 \mathrm{mmol} / \mathrm{l}$ ergaben eine Wiederfindung von durchschnittlich $99,5 \%(98,4$ bis $101,6 \%)$.

\section{Drift}

In 2 Versuchen über 30 Minuten entsprechend 40 Proben ergab sich jeweils eine Basisliniendrift von $0,00 \mathrm{mmol} / 1$ und eine Standarddrift von $+0,28 \mathrm{mmol} / 1$ $(5 \mathrm{mg} / \mathrm{dl})$ bei einer Konzentration von $11,4 \mathrm{mmol} / 1$ $(205 \mathrm{mg} / \mathrm{dl})$. Es sei hier besonders darauf hingewiesen, daß wir erst nach Einbau eines 340 nm-Filters auch in den Referenzkanal des Kolorimeters diese geringen Drifterscheinungen erreichten.

\section{Literatur}

1. Sadoff, H. L. (1966), in Methods in Enzymology (Colowick, S. P. \& Kaplan, N. O., ed.), Vol. 9, 103-107, Academic Press, New York.

2. Banauch, D., Brümmer, W., Ebeling, W., Metz, H., Rindfrey, H., Lang, H., Leybold, K. \& Rick, W. (1975), diese Z. 13, 101-107.

\section{Verschleppung}

Der Verschleppungskoeffizient nach Hjelm (5) wurde an wäßrigen Standardlösungen mit einer hohen Konzentration von $27,8 \mathrm{mmol} / \mathrm{l}$ und einer niedrigen von $2,78 \mathrm{mmol} / 1 \mathrm{Glucose}$ entsprechend den Angaben bei Haeckel (6) bestimmt. Von der höheren zur niedrigeren Konzentration beträgt der prozentuale Verschleppungskoeffizient $2,8 \%$ bei einer Probenfrequenz von $80 / \mathrm{h}$ und einer Waschzeit von 6 Sekunden, $2,4 \%$ bei einer Probenfrequenz von $80 / \mathrm{h}$ und einer Waschzeit von 8 Sekunden und $1,7 \%$ bei einer Probenfrequenz von $60 / \mathrm{h}$ und einer Waschzeit von 8 Sekunden. Das maximale Konzentrationsverhältnis zweier aufeinanderfolgender Proben sollte erfahrungsgemäß 3:1 nicht überschreiten. Anderenfalls empfiehit sich die Wiederholung der niedrigen Probe als Doppelbestimmung, wobei der zweite Wert Gültigkeit hat.

\section{Danksagung}

Wir danken Frau $H$. Thies für ihre sorgfältige Mitarbeit.

3. E. Merck AG. Darmstadt, Arbeitsanleitung für den Technicon AutoAnalyzer II, Stand April 1976.

4. Scholer, A. \& Pianezzi, A. (1976), diese Z. 14, 189-195.

5. Hjelm, H. (1968), Z. Anal. Chem. 243, 781-790.

6. Haeckel, R. \& Porth, A. J. (1972), diese Z. 10,91-94.

Dr. med. K. Kornmüller Dr. med. O. Müller-Plathe Zentrallaboratorium des Allgemeinen Krankenhauses Altona Paul-Ehrlich-Straße 1 2000 Hamburg 50 
